\title{
Les tuteurs des CAF : leurs représentations et les dispositifs qu'ils emploient
}

\section{HRONIQUE • Recherche étudiante}

\section{Problématique}

Les centres d'aide en français des cégeps (CAF) offrent divers services, dont le plus répandu est le tutorat (Nolet, 2019). Le tuteur, un cégépien doué en français et formé plus ou moins longuement, offre de l'aide à un autre étudiant, le tutoré. Les cégeps injectent des ressources humaines et financières dans le tutorat: des enseignants forment et encadrent les tuteurs, tandis que des professionnels et du personnel de soutien collaborent aux activités du CAF (Nolet, 2019).

Les rares recherches sur le tutorat dans les CAF portent sur les représentations des étudiants non tutorés (Cabot et Facchin, 2020) ou sur les effets du tutorat chez les tutorés (Gélinas, 2001). Des recherches menées au cégep et à l'université se penchent sur les pratiques générales des tuteurs, soit le type de soutien offert, la rétroaction donnée, etc. (Barrette, 2015; Désy, 1996). Or, à notre connaissance, aucune recherche ne se penche sur les dispositifs utilisés pour soutenir les tutorés en écriture (ex.: écriture guidée, dictée), alors que certains dispositifs favoriseraient plus que d'autres le développement de la compétence scripturale (Nadeau et Fisher, 2006). Il est donc nécessaire de mieux connaître les dispositifs utilisés par les tuteurs des CAF. Puisque les représentations d'une personne guident son action (Fortier et al., 2018), il convient également d'explorer les représentations des tuteurs. Ainsi, les questions de ce projet de maîtrise sont : comment les tuteurs des CAF soutiennentils le développement de la compétence scripturale des tutorés, et plus particulièrement, comment se représentent-ils leur rôle et quels sont les dispositifs à travers lesquels ils cherchent à soutenir leurs tutorés en écriture? 


\section{Cadre de référence}

Afin de répondre aux questions de recherche, il apparaît important de définir la représentation du rôle de tuteur ainsi que les dispositifs pédagogiques.

La représentation du rôle du tuteur renvoie à l'ensemble des opinions, des croyances et des connaissances qu'il entretient à propos des attentes qu'il croit devoir combler (rôle attendu), des pratiques qu'il dit adopter (rôle joué) ou de celles qu'il voudrait adopter (rôle souhaité) (Beauregard, 2006). Cette représentation relève de ses expériences (ex.: formation pour devenir tuteur, expériences scolaires), des attentes institutionnelles et sociales (ex. : attentes du CAF et des tutorés) et des rapports qu'il entretient avec ses expériences comme avec les attentes institutionnelles et sociales (Beauregard, 2006). Elle guide son action, notamment le choix de ses interventions pédagogiques (Fortier et al., 2018).

Un dispositif d'enseignement est un ensemble de moyens organisés dans le but qu'un apprenant réalise des apprentissages (Weiser, 2010). À l'instar de Weisser (2010), nous distinguons les dispositifs pédagogiques applicables peu importe la discipline, comme l'apprentissage coopératif ou l'enseignement magistral, des dispositifs didactiques qui visent l'enseignement et l'apprentissage d'une discipline particulière, comme le français ou les mathématiques. Ce second type de dispositifs puise dans les connaissances propres à la discipline enseignée. Selon Nadeau et Fisher (2006) ainsi que MontésinosGelet et al. (2018), il existe plusieurs dispositifs didactiques d'écriture, dont l'atelier de négociation graphique, les différentes formes de dictées, l'écriture modelée ou guidée, etc.

Au regard des deux conceptualisations exposées et puisque plusieurs facteurs peuvent influencer le choix des dispositifs, dont les représentations du tuteur, la recherche proposée poursuit les objectifs spécifiques suivants. D’abord, elle veut décrire la représentation du rôle de tuteurs ouvrant dans des CAF. Ensuite, elle veut décrire les dispositifs pédagogiques et didactiques privilégiés durant les rencontres de tutorat et comprendre ce qui mène à leurs choix.

\section{Méthodologie}

Pour atteindre ces objectifs, un devis mixte séquentiel explicatif a été retenu. Ce type de devis suppose deux phases de recherche et vise la compréhension générale d'un phénomène, mais aussi la perception de certains acteurs concernés dans le but d'affiner et d'approfondir cette compréhension générale (Briand et Larivière, 2014).

Durant la première phase, un questionnaire autoadministré portant sur les trois dimensions de la représentation des tuteurs (rôles attendu, joué et souhaité) et sur l'utilisation des dispositifs sera rempli par des tuteurs œuvrant dans des CAF $(n=30)$ de dix cégeps. Des analyses statistiques descriptives (moyennes, pourcentages, fréquences) seront réalisées pour documenter les représentations des tuteurs et les dispositifs employés. Elles permettront de cibler les éléments méritant d'être approfondis, nuancés ou complétés, et les participants auprès desquels il serait intéressant de recueillir de l'information supplémentaire. Les tuteurs recrutés pour la deuxième phase de collecte de données auront des profils différents. Ils pourraient avoir des représentations différentes de leur rôle ou privilégier différents dispositifs. Ainsi, durant la deuxième phase, le point de vue de ces tuteurs $(n=5)$ sera recueilli lors d'une entrevue semi-dirigée. Une analyse de contenu sera menée pour mieux comprendre et nuancer les résultats obtenus par le questionnaire.

2 - Formation et profession 29(1), 2021 
La collecte de données aura lieu tout au long de la session d'hiver 2021. Les résultats permettront de mieux connaître les services de tutorat des CAF. La diffusion des résultats aux enseignants des cégeps pourra servir, ultimement, à bonifier la formation des tuteurs.

\section{Références}

Barrette, C. (2015). Les conditions d'efficacité de l'aide par les pairs. Consortium d'animation sur la persévérance et la réussite en enseignement supérieur. http://www.capres.ca/wp-content/uploads/2015/05/Dossier final 22 mai 2015.pdf

Beauregard, F. (2006). Représentations sociales des parents et des enseignants de leurs rôles dans l'intégration scolaire d'un élève dysphasique en classe ordinaire au primaire. Revue des sciences de l'éducation, 32(3), 545-565. https://doi.org/ https://doi.org/10.7202/016276ar

Briand, C. et Larivière, N. (2014). Les méthodes de recherche mixtes : illustration d'une analyse des effets cliniques et fonctionnels d'un hôpital de jour psychiatrique. Dans M. Corbière (dir.), Méthodes qualitatives, quantitatives et mixtes : dans la recherche en sciences humaines, sociales et de la santé (p. 625-648). Presses de l'Université du Québec. http://ebookcentral.proquest.com/lib/umontreal-ebooks/detail.action?docID=4797068

Cabot, I. et Facchin, S. (2020). Identification des raisons de non-fréquentation des centres d'aide en français du postsecondaire au Québec. Revue internationale de pédagogie de l'enseignement supérieur, 36(1). https://doi.org/https://doi.org/10.4000/ripes.2406

Désy, J. (1996). Le tutorat par les pairs tel que perçu par les élèves. Cégep de Sainte-Foy. https://cdc.qc.ca/parea/714736-desy-tutorat-par-les-pairs-sainte-foy-PAREA-1996.pdf

Fortier, M.-P., Noël, I., Ramel, S. et Bergeron, G. (2018). Intégration scolaire, éducation inclusive et représentations des enseignants : de la formation initiale à la communauté éducative. Revue des sciences de l'éducation, 44(1), 12-39. https://doi.org/https://doi.org/10.7202/1054156ar

Gélinas, S. (2001). Impact d'un suivi au centre d'aide en français sur la performance scolaire et l'estime de soi d'étudiants éprouvant des difficultés en français écrit [mémoire de maîtrise, Université du Québec à Trois-Rivières]. Cognitio, http://depot-e.uqtr.ca/2571/1/000694354.pdf.

Montésinos-Gelet, I., Dupin de Saint-André, M. et Charron, A. (2018). Accompagnement des enseignants d'une école montréalaise quant au recours à des réseaux d'euvres littéraires pour soutenir la conscience linguistique en lecture et en écriture, (Rapport FRQSC, $n^{\circ}$ 2015-LC-187912). http://www.frqsc.gouv.qc.ca/documents/11326/2510085/I.MontesinosGelet rapport conscience-linguistique-lecture-ecriture.pdf.pdf/280fafd5-e395-4567-9b28-1c39ef2f2b6c

Nadeau, M. et Fisher, C. (2006). La grammaire nouvelle : la comprendre et l'enseigner. Gaëtan Morin.

Nolet, M.-J. (2019). Les CAF en chiffres : faits saillants d'une enquête sur la situation des centres d'aide en français. Correspondance, 24(9). http://correspo.ccdmd.qc.ca/index.php/document/les-caf-en-chiffres-faits-saillants-duneenquete-sur-la-situation-des-centres-daide-en-francais/

\section{Pour citer cet article}

Thomas, V. (2021). Les tuteurs des CAF : leurs représentations et les dispositifs qu'ils emploient [chronique]. Formation et profession, 29(1), 1-3. http://dx.doi.org/10.18162/fp.2021.a220 